\title{
Research on energy storage depth planning of AC I DC distribution network based on toughness enhancement technology
}

\author{
Yidan $\mathrm{Hu}^{1}$ \\ ${ }^{1}$ Nanjing University of Science and Technology ZiJin College, Nanjing, Jiangsu 210023, China
}

\begin{abstract}
At present, due to the abnormal objective function setting result of AC / DC distribution network energy storage depth planning method, the operation cost of power supply network has been increased frequently. Therefore, the energy storage depth planning method of AC / DC distribution network based on toughness enhancement technology is designed. The minimum peak valley difference, maximum load rate and minimum load change are taken as the energy storage depth planning objectives of $\mathrm{AC} / \mathrm{DC}$ distribution network. The economic mathematical model of distribution network planning is constructed by using toughness enhancement technology and objective function to complete the in-depth planning of distribution network. So far, the energy storage depth planning method of AC / DC distribution network based on toughness enhancement technology has been completed. Simulation experiment has been constructed. Through the comparison, it can be seen that this method has better effect than the original method, possessing stronger control ability for operation components.
\end{abstract}

\section{Introduction}

$\mathrm{AC}$ and DC have always been the main forms of electric energy in the distribution network. During the construction of the distribution network, both have experienced long-term competition. With the application of a series of $\mathrm{AC}$ equipment such as transformers and three-phase motors, AC once replaced DC power. Nowadays, most of the current forms used in medium and low-voltage distribution networks are AC [1-2]. However, through the references, it is found that there will be corresponding problems in the use of AC. For example, in the field of high-voltage transmission, the effect of AC is not as good as that of DC. Therefore, DC occupies a place in the high-voltage transmission network. With the development of distribution network, DC power supply and AC power supply have made significant changes. As the power electronic technology continuously progresses, the cost of DC / AC conversion equipment has decreased. Energy storage in $\mathrm{AC} / \mathrm{DC}$ power distribution network has become a hot issue of concern.

At present, the $\mathrm{AC} / \mathrm{DC}$ hybrid distribution network is still in the stage of theoretical research, and its grid topology and control strategy have been the focus of research at home and abroad. In reference ${ }^{[3]}$, three topology schemes of AC / DC hybrid microgrid are proposed and compared, and the design principles of $\mathrm{AC}$ / DC hybrid microgrid topology are summarized; In reference $\left.{ }^{[4}\right]$, a double droop control strategy is proposed for $\mathrm{AC} / \mathrm{DC}$ microgrid interface converter, which combines active power frequency droop control on $\mathrm{AC}$ side and active power voltage droop control on DC side to realize coordinated operation of AC / DC microgrid; Reference ${ }^{[5]}$ considers that in the grid connected state, $\mathrm{AC} / \mathrm{DC}$ hybrid microgrid should give priority to the use of energy storage to balance the system power, so as to reduce the power exchange between the system and the main network; similar to the reference ${ }^{[5]}$, the reference [6] thinks that whether the grid connected or the isolated grid, the DC microgrid energy storage battery should always be under constant voltage control, and the interface converter should be coordinated to suppress the power fluctuation of the system.

Therefore, in this design, the toughness enhancement technology is used to optimize the shortcomings of the original method, and the AC / DC distribution network energy storage depth planning method based on the toughness enhancement technology is designed, providing a new direction for the development of distribution network.

"wuzhilxy@163.com 


\section{Design of energy storage depth planning method for AC I DC distribution network based on toughness enhancement technology}

\subsection{Setting the objective function of energy storage planning for AC / DC distribution network}

In this design, the minimum peak valley difference, maximum load rate ${ }^{[6-7]}$ and minimum load change are taken as the energy storage depth planning objectives of $\mathrm{AC} / \mathrm{DC}$ distribution network. Therefore, the objective function of AC / DC distribution network energy storage planning is set as three parts.

Minimum peak valley difference: this objective function is used to control the net load value of the grid after energy storage discharge. The specific calculation formula is as follows:

$$
P_{i}=\sum_{n=1}^{N_{1}} p_{1}-\sum_{n=1}^{N_{2}} p_{2}-\sum_{n=1}^{N_{3}} p_{3}+\sum_{n=1}^{N_{4}} p_{4}
$$

In the above formula, $N_{1}$ represents the number of load bearing at the grid bus. $N_{2}$ represents the number of PV installed at the grid bus. $N_{3}$ represents the number of exhaust devices installed at the grid bus. $N_{4}$ represents the number of energy storage devices installed at the grid bus. $p_{1}$ represents the load value in the power grid at the time of $n . p_{2}$ represents the output value of $\mathrm{PV}{ }^{[8-10]}$ in the grid at the time of $n . p_{3}$ represents the output value of exhaust equipment in the grid at the time of $n . p_{4}$ represents the charging and discharging power value of the grid at the time of $n$. When the charging and discharging power is positive, the energy storage is equal to the corresponding value. Otherwise, the energy storage is equivalent to the power supply for discharging. According to the above formula, the net peak valley difference can be obtained. The specific formula is as follows ${ }^{[11]}$ :

$$
\min \left(\max \left(p_{1}, p_{2}, \ldots, p_{n}\right)-\min \left(p_{1}, p_{2}, \ldots, p_{n}\right)\right)
$$

In the above formula, $p_{1}, p_{2}, \ldots, p_{n}$ represents the net load value of the power grid at different times. In this research, the time unit is set as day. Therefore, 24 hours is taken as a unit in the calculation of time.

Maximum of net load rate: this objective function represents the ratio between the mean value of net load value and the peak value of net load value, which can be expressed as follows ${ }^{[12]}$ :

$$
M=\frac{\frac{1}{t} \sum_{t=1}^{t} P_{i}}{\max \left(p_{1}, p_{2}, \ldots, p_{n}\right)}
$$

Using this formula, the above objective function can be calculated and the corresponding value can be obtained $^{[13]}$.

Minimum net load change: this objective function can represent the difference between the net load values at adjacent times, and the energy storage effect of the power grid can be controlled by reducing the load variation. This objective function can be expressed as follows by formula ${ }^{[14]}$ :

$$
I=\min \sum_{t=1}^{T}\left|P_{i}-P_{i-1}\right|
$$

In the above formula, $P_{i}$ represents the net load value at the time of $i . P_{i-1}$ represents the net load value at the previous time of $P_{i}{ }^{[15]}$. Through the above three groups of objective functions, the energy storage mode of power grid is optimized.

\subsection{Construction of distribution network planning mathematical model}

According to the above objective function, the mathematical model of distribution network planning is constructed as an economic model ${ }^{[16]}$. At the same time, the data model is optimized by toughness enhancement technology, and the specific processing process is as follows ${ }^{[17]}$.

In the process of model design, the investment cost, operation cost and equipment maintenance cost ${ }^{[12-13]}$ are taken as one of the main reference contents of the model, and the toughness enhancement technology is used to process the three parts of data. Under the premise of system decision-making permission, this constraint condition is compromised with the objective function, and the following formula is obtained ${ }^{[18]}$.

$$
\left\{\begin{array}{l}
\min f(x, \alpha) \\
\text { s.t. }\left\{\begin{array}{l}
Y_{i}\left\{h_{i}(x, \alpha) \leq 0\right\} \geq \beta \\
G(x, \alpha) \leq 0
\end{array}\right.
\end{array}\right.
$$

In the above formula, $\alpha$ represents the random coefficient in the objective function of the distribution network. $f(x, \alpha)$ represents the optimized objective function. $Y_{i}\left\{h_{i}(x, \alpha) \leq 0\right\}$ represents the constraint condition of the planning model. $h_{i}(x, \alpha)$ represents the chance constraint for the model establishment ${ }^{[19-20]} \beta$ represents the confidence level of the chance constraint condition. The toughness enhancement technology is used to optimize this part of the model. $G(x, \alpha) \leq 0$ is the rigid condition of this model ${ }^{[21]}$, which must be met to achieve the effect of this model. By processing this model, the energy storage planning of distribution network can be realized on the premise of ensuring the economic efficiency of distribution network. So far, the energy storage depth planning method of AC / DC distribution network based on toughness enhancement technology has been completed ${ }^{[22-23]}$. 


\section{Simulation experiment analysis}

\subsection{Design of experimental scheme}

In this experiment, the design method and traditional method are used to plan the selected distribution network segment. In order to compare the difference between the design method and the traditional method, the change of operation cost and the stability of distribution network after planning are taken as the experimental objects. In the process of the experiment, the experimental environment is set as two seasons, winter and summer. Each season is set as three months to obtain the experimental results and analyze the differences in the use of various methods.

\subsection{Analysis of experimental results}

TABLE I. EXPERIMENTAL RESULTS OF DISTRIBUTION NETWORK OPERATION COST CHANGE AFTER PLANNING

\begin{tabular}{|c|c|c|c|c|}
\hline Season & Experimental month & $\begin{array}{l}\text { Operating cost } \\
\text { change rate of } \\
\text { design method } \\
\text { in this paper } / \%\end{array}$ & $\begin{array}{c}\text { Traditional } \\
\text { method } 1 \\
\text { operating cost } \\
\text { change rate } / \%\end{array}$ & $\begin{array}{c}\text { Traditional } \\
\text { method } 2 \\
\text { operating cost } \\
\text { change rate } / \%\end{array}$ \\
\hline \multirow[t]{3}{*}{ winter } & 1 & -2.75 & 5.24 & 6.45 \\
\hline & 2 & -3.24 & 6.47 & 7.15 \\
\hline & 3 & -2.54 & 8.24 & 6.25 \\
\hline \multirow[t]{3}{*}{ summer } & 1 & -2.64 & 3.41 & 4.21 \\
\hline & 2 & -3.84 & 4.56 & 4.35 \\
\hline & 3 & -1.15 & 3.45 & 4.51 \\
\hline
\end{tabular}

network after use, showing a positive growth state in

From the above experimental results, it can be seen that the operation cost of distribution network shows a significant decline after using the design method in this paper. In the months with large power consumption in winter and summer, the operation cost fluctuation of distribution network is small and tends to be flat. It can be seen that the operation cost of distribution network can be effectively controlled and the economic cost can be saved by using the design method in this paper. Compared with the design method in this paper, the original method 1 and the original method 2 have a lower ability to control the operation cost of distribution many months. In the months with large power consumption in winter and summer, the operation cost of distribution network is not limited. Therefore, these two methods can not meet the needs of people in the daily application process, causing certain economic losses and waste of resources. According to the above analysis results, the design method in this paper can effectively improve the economy of distribution network, provide more economical and affordable electricity for people, and promote the implementation of the concept of sustainable development.

TABLE II. POWER SUPPLY STABILITY TEST RESULTS OF DISTRIBUTION NETWORK AFTER PLANNING

\begin{tabular}{ccccc}
\hline season & Experimental month & $\begin{array}{c}\text { Power supply } \\
\text { stability of } \\
\text { distribution } \\
\text { network is designed } \\
\text { in this paper } / \%\end{array}$ & $\begin{array}{c}\text { Traditional method } \\
\text { 1 power supply } \\
\text { stability of } \\
\text { distribution } \\
\text { network } / \%\end{array}$ & $\begin{array}{c}\text { Traditional method } \\
\text { 1 power supply } \\
\text { stability of } \\
\text { distribution } \\
\text { network } / \%\end{array}$ \\
\hline winter & 1 & 97.25 & 94.24 & 93.14 \\
summer & 2 & 97.85 & 93.15 & 93.64 \\
& 3 & 97.14 & 93.17 & 93.41 \\
& 1 & 97.64 & 94.19 & 94.51 \\
& 3 & 98.01 & 95.15 & 93.15 \\
\hline
\end{tabular}

Analysis of the above experimental data shows that after using the design method in this paper, the power supply stability of the distribution network is relatively high. In a variety of experimental environments, there is no collapse of the distribution network or excessive current in the distribution network. It can be seen that the design method in this paper can effectively control the power transmission and power supply in the power grid. Using this method in winter and summer with large power supply can effectively improve the satisfaction of users and ensure their normal power consumption. Therefore, through the above experimental results, the design method in this paper is better than the traditional method.

According to the above experimental results, the design method in this paper has certain reliability in daily use, and its use effect is obviously better than that of the traditional method. In the future distribution network optimization process, this method can be used to improve the performance of distribution network. 


\section{Conclusion}

In this research, the $\mathrm{AC} / \mathrm{DC}$ distribution network energy storage is deeply planned. Due to the time and technology constraints, there are corresponding deficiencies in this research. In the future research, combined with the existing research results and the development trend of AC / DC distribution network, some deficiencies in this paper will be optimized, so as to provide theoretical basis for the use and development of AC / DC distribution network, promote the development process of China's distribution network, and realize the reform of energy structure in China.

\section{References}

1. LI W, CHEN H D, CUI Z T, et al. A configuration method of energy storage device in DC distribution network based on discrete Fourier spectrum analysis[J]. Power System Protection and Control,2019,47(07):92-98.

2. FU S Q, GAO Y, CHEN X Y, et al. Research and Project Practice on AC and DC Distribution Network Based on Flexible Substations[J]. Electric Power Construction,2018,39(05):46-55.

3. SHAO Z, ZOU X S, YUAN X F, et al. Review on energy storage application research in the context of flexible distribution network[J]. Electrical Measurement \& Instrumentation,2020,57(03):86-93.

4. SHEN M, ZHANG X B. Research on VSC-LVDC with DC Energy Storage to Improve the Stability of Microgrid Connected to Grid Operation[J]. Journal of Northwestern Polytechnical University,2018,36(03):528-535.

5. A Y L, A B F, A G L, et al. Optimal distributed generation planning in active distribution networks considering integration of energy storage ScienceDirect[J]. Applied Energy, 2018, 210:10731081.

6. Ma J, Qiao F. A Coordinated Voltage/Var Control in a Hybrid AC/DC Distribution Network[J]. IET Generation Transmission \& Distribution, 2020, 14(11).

7. José M. Maza-Ortega, Mauricio J M, Manuel Barragán-Villarejo, et al. Ancillary Services in Hybrid AC/DC Low Voltage Distribution Networks[J]. Energies, 2019, 12(19):3591-3601.

8. ZHU Z S, LIU D C, LIAO Q F, et al. Optimal Power Scheduling for a Medium Voltage AC/DC Hybrid Distribution Network[J]. Sustainability, 2018, 10(2):318-327.

9. Liu Q, Wang S, Ji X, et al. Power sensitivity models with wide adaptability in active distribution networks considering loops and DC networks[J]. International Transactions on Electrical Energy Systems, 2020, 30.

10. Wang, Li, Zhao, et al. Reduced-Order DC Terminal Dynamic Model for Multi-Port AC-DC Power
Electronic Transformer[J]. Energies, 2019, 12(11):2130.

11. Gerber D L, Vossos V, Feng W, et al. A simulationbased efficiency comparison of $\mathrm{AC}$ and $\mathrm{DC}$ power distribution networks in commercial buildings $[\mathrm{J}]$. Applied Energy, 2018, 210(jan.15):1167-1187.

12. Xie B, Wang J, Jin Y, et al. Power Distribution Control Scheme for a Three-phase Interleaved DC/DC Converter in the Charging and Discharging Processes of a Battery Energy Storage System[J]. JOURNAL OF POWER ELECTRONICS, 2018, 18(4):1211-1222.

13. Bhattacharya A, Kharoufeh J P , Zeng B . Structured Storage Policies for Energy Distribution Networks[J]. IIE Transactions, 2018, 50(8):683-698.

14. Sperstad I, Korpås, Magnus. Energy Storage Scheduling in Distribution Systems Considering Wind and Photovoltaic Generation Uncertainties[J]. Energies, 2019, 12(7).

15. Zheng Y, Meng K, Luo F, et al. Optimal Integration of Mobile/Stationary Battery Energy Storage Systems in Distribution systems with Renewables[J]. IET Renewable Power Generation, 2018, 12(10):258-269.

16. Li, He, Fang, et al. Flexible Voltage Control Strategy Considering Distributed Energy Storages for DC Distribution Network[J]. IEEE Transactions on Smart Grid, 2019, 10(1):163-172.

17. Li Y, Pei X, Yang M, et al. An Advanced Fault Control of Transformerless Modular Multilevel Converters in AC/DC Hybrid Distribution Networks under the Single-phase Grounding Fault[J]. IEEE Transactions on Power Delivery, 2020, PP(99):1-1.

18. Khan M O, Zaman S, Noh C H, et al. A Load Flow Analysis for AC/DC Hybrid Distribution Network Incorporated with Distributed Energy Resources for Different Grid Scenarios[J]. Energies, 2018, 11(2):367-378.

19. Ye C, Miao S, Li Y, et al. Hierarchical Scheduling Scheme for AC/DC Hybrid Active Distribution Network Based on Multi-Stakeholders[J]. Energies, 2018, 11(10).

20. Zhang L, Chen Y, Shen C, et al. Optimal Configuration of Hybrid ACDC Urban Distribution Networks for High Penetration Renewable Energy[J]. IET Generation, Transmission \& Distribution, 2018, 12(20):4499-4506.

21. FU Y, ZHANG Z Q, LI Z K. Energy Management for Hybrid AC/DC Distribution System With Microgrid Clusters Using Non-Cooperative Game Theory and Robust Optimization[J]. IEEE Transactions on Smart Grid, 2019, 11(2):1510-1525.

22. A H Q, A W G, B J P, et al. Multi-intervaluncertainty constrained robust dispatch for AC/DC hybrid microgrids with dynamic energy storage degradation[J]. Applied Energy, 2018, 228:205-214.

23. Judge $P$ D, Green $T$ C. Modular Multilevel Converter With Partially Rated Integrated Energy 
Storage Suitable for Frequency Support and Ancillary Service Provision[J]. IEEE Transactions on Power Delivery, 2019, 34(1):208-219. 